\title{
La geometría del códice y su semiótica
}

\section{The codex geometry and its semiotics}

\author{
Ana Belén SÁNCHEZ PRIETO \\ Universidad Complutense de Madrid, Facultad de Ciencias de la Documentación \\ anabelsa@pdi.ucm.es
}

Recibido: 06/11/2012

Aprobado: 06/12/2012

Resumen: La impaginación o mise en page de un libro consiste en un diseño realizado mediante diversos rectángulos que normalmente guardan entre sí ciertas proporciones definidas. En la actualidad los tamaños y proporciones de estos rectángulos están determinados por razones prácticas y estéticas, pero en la Edad Media la Geometría estaba considerada como un medio de meditación metafísica. Este artículo considera posibles interpretaciones metafísicas para las medidas y los rectángulos proporcionales según aparecen en muchos manuscritos medievales.

Palabras Clave: Codicología, impaginación, mise en page, superficies armónicas, geometría sagrada.

Abstract: The page layout of a book is a design made of various rectangles that normally keep certain proportions. In present times the size and proportions of these rectangles are determined by practical and esthetical causes, but in the Middle Ages Geometry was known to be considered as a mean of metaphysical meditation. This article deals with the possible metaphysical interpretation of measures and proportional rectangles as they appear in mediaeval manuscripts.

Key Words: Codicology, page layout, mise en page, harmonical surfaces, sacred geometry.

Sumario: 1. Impaginación o mise en page. 2. Armonía, equilibrio y proporción. 3. "Geometría teológica". 4. Empleo de algunas superficies armónicas en la mise en page y propuesta de interpretación. 5. Interpretación de los valores absolutos de los números. 5. Conclusiones. Fuentes y Bibliografía.

\section{Impaginación o mise en page}

La geometría de un libro está constituida por una serie de rectángulos superpuestos unos a otros. Si se considera el libro abierto, en un diseño básico, estos rectángulos son: a) La doble página, b) cada una de las páginas que componen la doble página, c) las cajas de escritura (a línea seguida, o a doble -o múltiplecolumna), d) cada uno de los márgenes (ver Fig. 1). Si el diseño es más complicado, puede existir además una caja de escritura añadida, reservada para notas o comentarios, y en el libro impreso actual hay también espacios destinados a los encabezados y pies de página. 


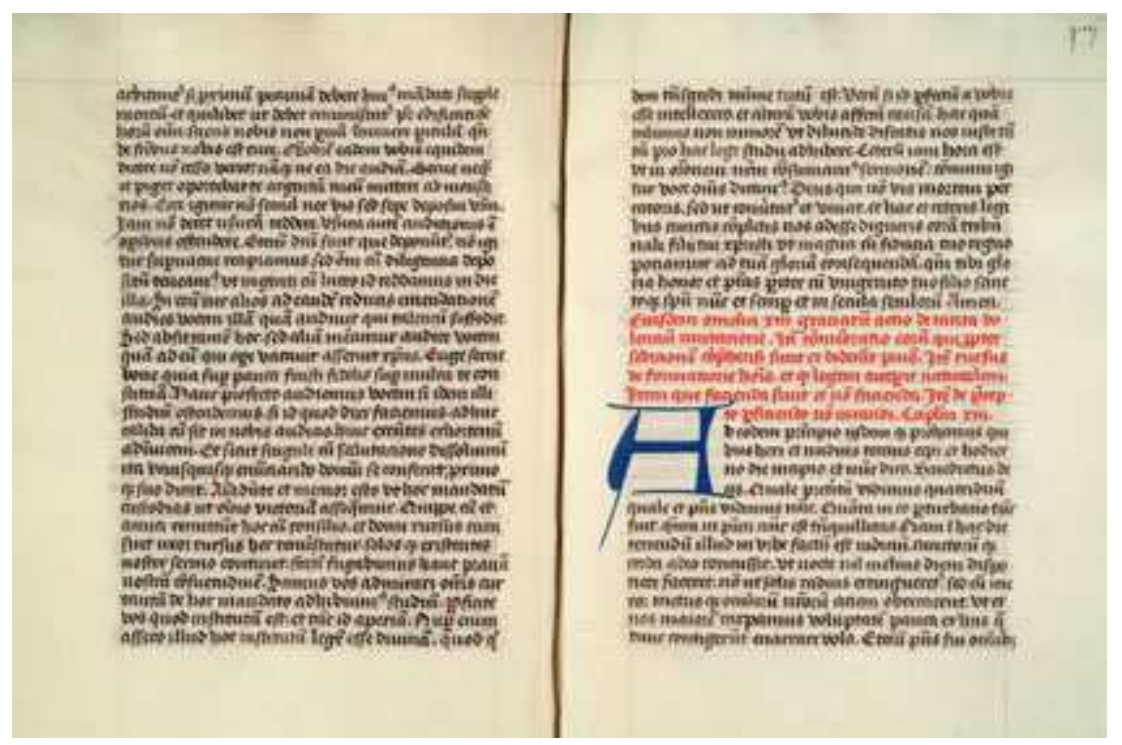

Fig. 1. Rouse MS 34. Homilías de san Juan Crisóstomo (Países Bajos, s. XV). (http://unitproj.library.ucla.edu/special/rouse/nether2.htm)

Pueden verse claramente los siguientes rectángulos: a), b), c) y d).

El copista ha delimitado la caja de escritura mediante líneas de justificación perfectamente apreciables.

En codicología el conjunto de operaciones por la cual el copista planifica la dimensión de su doble página y la posición y disposición del texto sobre ella recibe el nombre de impaginación ${ }^{1}$ o mise en page. Independientemente de la complejidad del diseño plasmado sobre la página, la mise en page siempre consiste en un cierto número de líneas rectas (reales o imaginarias), paralelas y perpendiculares entre sí y a los bordes de la página, cuya finalidad es separar los espacios destinados a la escritura de los espacios destinados a quedar en blanco o a recibir ornamentación.

\section{Armonía, equilibrio y proporción}

El tamaño y la relación de estos rectángulos nunca son arbitrarios, sino que están dictados bien por razones prácticas, bien por el valor estético o simbólico que puedan tener en el mundo cultural en que hayan sido diseñados. Si en la actualidad, el tamaño y las proporciones de esos rectángulos están determinados por las condiciones de legibilidad, los tamaños estándar del papel y el precio final del libro, en la época del libro manuscrito en pergamino, aparentemente no existe otra limitación que el tamaño de la piel, el uso que pretenda darse a cada manuscrito concreto y el capricho del artesano, y por lo tanto cualesquier combinaciones deberían ser posibles. Sin embargo, lo cierto es que de todas esas casi infinitas teóricas posibilidades de combinación, en la práctica se repiten una y otra vez las mismas proporciones, que forman un número relativamente pequeño.

Y casi todas estas proporciones repetidas una y otra vez siguen reglas matemáticas. Algunas de ellas pueden calificarse como "racionales", pues siguen proporciones "racionales": 1:2, 2:3, 3:4, 5:8 y 5:9 son las más corrientes. Otras son

\footnotetext{
${ }^{1}$ Elisa RUIZ GARCÍA, Introducción a la Codicología, Madrid, Fundación Germán Sánchez Ruipérez, 2002, pp. 179-180. 
"irracionales", en cuanto que están regidas geométricamente por números irracionales: 1:Phi (regla de oro, 1:618..., de la cual 5:8 es una aproximación), $1: \sqrt{2}, 1: \sqrt{ } 3,1: \sqrt{5}, 1: 1,538 \ldots$ (rectángulo pentagonal)... (ver Fig. 2). Por supuesto, también se hallan libros que aparentemente no siguen ninguna de las proporciones explicables geométricamente; estas superficies han sido denominadas por Tschichold "arbitrarias"

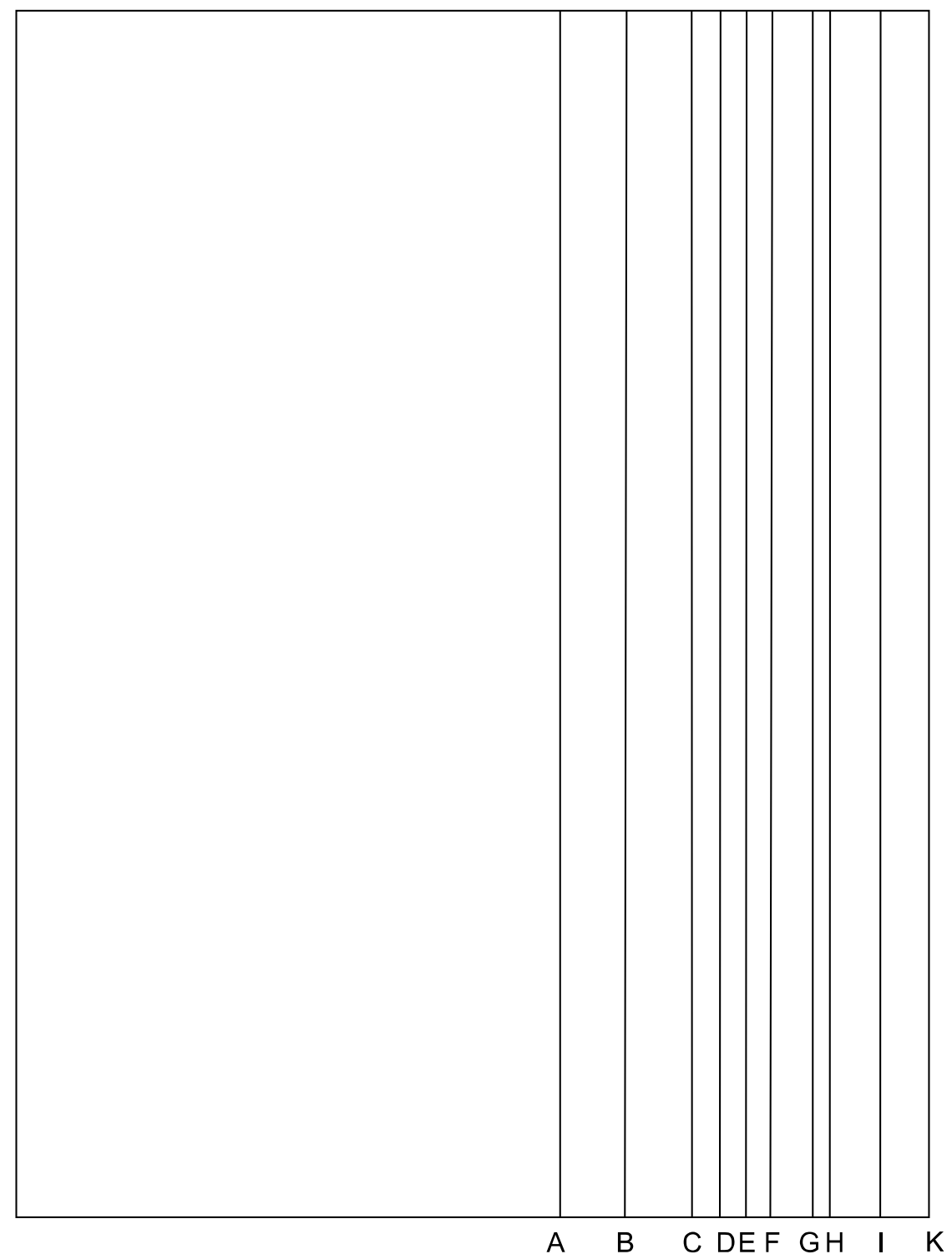

Fig. 2. Distintos rectángulos con proporciones matemáticas.

(Tomado de Tschichold, "Consistent correlation..”, p. 59).
A: $1: \sqrt{ } 5$
B: $1: 2$
C: 5:9
D: $1: \sqrt{ } 3$
E: $3: 5$
F:1:Phi (dorada proporción)

G: 1:358 (rectángulo pentagonal) H: 2:3 I: 1:V2 (rectángulo de proporción creciente)

K: 3:4 (rectángulo de Pitágoras)

${ }^{2}$ Jan TSCHICHOLD, "Non-Arbitrary Proportions of Page and Type Area", en A.S. OSLEY (ed.), Calligraphy and Paleography. Essays presented to Alfred Fairbank, London, Faber and Faber, 1965, pp. 179-191, cit.pp. 179-180, e id. "Correlation between book page and type area", en Jan TSCHICHOLD, The form of the book. Essays on the morality of good design, Vancouver, Hartley \& Marks, 1991, pp. 36-63, cit. pp. 37-38. 
Para comprender esta recurrencia hay que tener en cuenta que una página escrita es el resultado de una serie de exigencias funcionales (legibilidad fundamentalmente, pero también otras como el destino final del manuscrito como producto de exposición o lectura privada, producto de lujo y ostentación o estrictamente funcional...), pero además es fruto de un desarrollo cultural previo. Este desarrollo cultural explica por ejemplo, por qué Gutenberg prefiere una página en proporción 2:3, o que durante el Renacimiento rara vez se utilice la proporción 1: $\sqrt{2}$, que había sido bastante corriente durante la Edad Media ${ }^{3}$.

Igual que en el terreno paleográfico cada lengua o asunto manifiesta unas determinadas preferencias en la elección de los tipos de letra, la elección del diseño de página parece seguir unas pautas similares. Por ejemplo, Carla Bozzolo, Dominique Coq, Denis Muzerelle y Ezio Ornato han encontrado patrones de uso diferentes en los manuscritos franceses de finales de la Edad Media, dependiendo de que hayan sido escritos en latín o en francés, por lo que distinguen entre una "page savante" y una "page vulgaire": en tanto que en los manuscritos latinos la relación entre anchura y altura es de $1: \sqrt{2}$ (esto es, entorno a 0,707), que es precisamente una de las proporciones geométricas identificadas por Tschichold, los manuscritos franceses tienden a tener una página algo más larga $(1: 1,423$, o sea, 0,7022 de media). Y la diferencia es aún más evidente si se considera la proporción de la caja de escritura: 0,682 de media para los manuscritos latinos frente a 0,727 para los franceses. Además, en los manuscritos franceses la caja de escritura no se ensancha cuando la disposición de texto tiene lugar a doble columna (0,691 para los manuscritos latinos, 0,726 para los franceses, donde además el espacio entre ambas columnas es algo más ancho, lo cual indica que aquí el copista no estaba interesado en compensar el espacio de escritura que se pierde en el intercolumnio haciendo los márgenes algo más estrechos). En efecto, en el caso de los manuscritos en lengua vernácula, los artesanos pueden expresarse más libremente, al hallarse más libres de constricciones culturales, y pueden utilizar en sus diseños medidas más próximas a las dimensiones naturales de las pieles o las formas ${ }^{4}$ comúnmente utilizadas para la fabricación del papel ${ }^{5}$.

La relación entre las dimensiones de la página y las de la caja de escritura determina la anchura y altura de los márgenes. Pocas veces los márgenes han recibido atención ${ }^{6}$. La práctica habitual de los catalogadores es un reflejo de la tendencia espontánea a subordinar la parte "blanca" de la página a la parte escrita, ya que es esta última parte la destinada a transportar la información. Desde esa perspectiva, los márgenes son considerados como "no-texto", y sobre todo si

\footnotetext{
${ }^{3}$ TSCHICHOLD, "Correlation...," p. 41.

${ }^{4} \mathrm{La}$ forma es un molde rectangular formado por una especie de rejilla de hilos metálicos dispuestos sobre un bastidor de madera que sirve para sujetarla. Su finalidad es recoger las materias que flotan en suspensión en la pasta de papel, de modo que sobre el entramado se deposita una delgada película que, una vez secada y encolada, formará la hoja de papel.

${ }^{5}$ Carla BOZZOLO; Dominique COQ; Denis MUZERELlE \& Ezio ORNATO, "Page savante, page vulgaire: Étude comparative de la mise en page des livres en latin et en français écrits ou imprimés en France au XVe siècle", en La face cachée du livre médiéval. L'histoire du livre vue par Ezio Ornato, ses amis et ses collègues, Roma, Viella, 1997, pp., 509-517, cit. p. 513.

${ }^{6}$ Una excepción es Curt F. BÜHLER, "The margins in mediaeval books", The Papers of the Bibliographica Society of America 40/1 (1946), pp. 32-42.
} 
carecen de decoración son "tierra de nadie", y a lo sumo serán considerados como portadores de glosas u otros signos de lectura, pero nunca como parte del diseño de la página.

No se hallaban en tal caso los maestros de escriptorio medievales, o al menos eso da a entender una glosa hallada en el folio 2 del Ms. Paris, BN de France, Lat. $11884^{8}$, donde las proporciones de los márgenes en relación al tamaño total de la página y al de la caja de escritura aparecen cuidadosamente indicadas:

Taliter debet fieri quaternionis forma, quinta parte longitudinis, quarta latitudinis. Quintam partem da inferiori vel anteriori margini, et ipsam quintam partem diuide in .III. et dabis .II. superiori. subtracta .I. Rursus ipsas .II. partes divide in tres, dabisque duas posteriori margini subtrahendo unam. Huic compar erit si media interfuerit. Lineas vero iuxta rationem scripturae divides, quia maior scriptura latioribus, minor autem strictioribus lineis indiget.

Según esta receta, la superficie total de la página (100\% x 80\%) queda dividida en:

- Caja de escritura: $66^{2 / 3} \%$ x $51^{1 / 9} \%$

- Margen inferior: $20 \%$

- Margen superior: $13^{1 / 3} \%$

- Margen exterior: $20 \%$

- Margen interior: $8^{8 / 9} \%$

Por supuesto, no todos los manuscritos medievales seguían necesariamente esta guía, y dada la falta de datos en los catálogos actuales no se puede ofrecer ningún tipo de tendencia. Pero incluso si las medidas de los márgenes aparecieran en los catálogos, habría que tener en cuenta que con frecuencia las medidas originales serían algo más grandes, sobre todo en los casos (que son la mayoría) en los que los manuscritos en cuestión hayan sido reencuadernados, ya que los encuadernadores acostumbraban a igualar los cantos de los libros cizallándolos o guillotinándolos, por lo cual es fácil que cada encuadernación se haya llevado al menos uno o dos milímetros por cada lado.

Aparte de las medidas de los márgenes, una característica casi general tanto de los libros manuscritos como de los impresos es que los márgenes inferior y exterior son más anchos que el superior y sobre todo que el interior. Es decir, la caja de escritura no está centrada en la página, sino desviada en dirección del ángulo superior interior. Por supuesto, hay razones prácticas para esta disposición: los

7 Marilena MANIACI \& Ezio ORNATO, "Intorno al testo. Il ruolo dei margini nell'impaginazione dei manoscritti greci e latini", Nuovi Annali della Scuola Speciale per Archivisti e Bibliotecari 9 (1995), pp. 175-194. Republ. en La face cachée du livre médiéval. L'histoire du livre vue par Ezio Ornato, ses amis et ses collègues, Rome, Viella, 1997, pp. 457-471, cit. pp. 457458.

${ }^{8}$ Fue publicada por Edward K. RAND en su Studies in the Script of Tours, II. The Earliest Book of Tours, Cambridge, Mass., The Mediaeval Academy of America, 1934, p. 88, y la reproduce también Bühler en el artículo citado, p. 36. 
márgenes interiores, al contrario que los demás, nunca sufrirán disminuciones causadas por encuadernadores, sin duda el maestro de escriptorio tenía también en cuenta (al menos en los libros de tamaño reducido) el hecho de que muchas veces los bordes de la piel (que necesariamente caen en los bordes de la página) producen un pergamino de peor calidad, en el que es más difícil escribir, por no mencionar el que el lector tiende a sostener el libro en sus manos por la parte inferior y externa. Pero más allá de los aspectos prácticos, el efecto visual que produce esta disposición es especular, y el lector percibe inmediatamente la simetría de las dos páginas del libro abierto, que de este modo se relacionan visualmente entre sí mucho más estrechamente que si ambas cajas de escritura estuvieran dispuestas sin más en el centro de la página.

Además, dentro de la tendencia general que acabamos de mencionar, se verifica la existencia de otras dos tendencias en la "geometría de los márgenes", pues el margen inferior tiende a tener la misma anchura que el margen exterior, y el superior la misma que el interior. $Y$ hablamos de tendencias (en plural) y no tendencia, porque, si bien nada impide en teoría que ambas igualdades tengan lugar simultáneamente, en la práctica es raro encontrarlas juntas, a pesar de que por separado se hallan en áreas y períodos de uso bien definidos ${ }^{9}$.

En definitiva, considerando en conjunto los aspectos geométricos de la mise en page, la página se define como un conjunto equilibrado de rectángulos (al menos en un libro bien diseñado). Y ese equilibrio está determinado por la extensión respectiva de los rectángulos y la armonía entre sus proporciones.

Este equilibrio y armonía se perciben instintivamente, y la razón parece ser fisiológica: la estimación que realiza el ojo de las longitudes relativas de los lados adyacentes de un rectángulo (A-B, A-D) es en última instancia reducible a la medida instintiva de la duración relativa de dos intervalos de tiempo. Lo que se aprecia a nivel subconsciente es la ratio del tiempo que requiere la línea de visión para pasar de A a B y de A a D, y estos intervalos son instintivamente medidos por los relojes corporales internos. Mientras que el foco de visión pasa de A a B, un cierto número de impulsos nerviosos pasa a través de los nervios ópticos en un período de tiempo proporcional al esfuerzo muscular del globo ocular. La experiencia armoniosa que se percibe cuando concurren ciertas longitudes obedece, pues, en última instancia a la misma razón por la cual ciertos intervalos musicales son percibidos como más armoniosos, cuando son reducibles a la proporción de números enteros pequeños, y son exactamente la octava (ratio de la frecuencia 2:1), tercera mayor $(5: 4)$ y sexta mayor $(8: 5)^{10}$.

\section{3. "Geometría teológica"}

Ahora bien, en el mundo antiguo y medieval la geometría es a menudo utilizada como una metáfora del orden universal y los números poseen un significado místico añadido a su función de indicar la cantidad. Por ejemplo, el uno no es

\footnotetext{
${ }^{9}$ Marilena MANIACI \& Ezio ORNATO, "Intorno al testo", p. 469.

${ }^{10}$ H. E. HUNTLEY, The Divine Proportion. A Study of Mathematical Beauty, New York, Dover Publications, 1970, pp. 52-56
} 
propiamente un número, sino el principio de los números ${ }^{11}$, y contiene en sí todas las cosas, y por eso es el símbolo de Dios Creador. El propio Dios Creador es descrito como un geómetra:

Cuando colocaba los cielos, allí estaba yo (i.e, la Sabiduría)

cuando trazaba la bóveda sobre la superficie del océano (Prov 8:27).

Y la Biblia afirma:

Pero tú regulaste todo con medida, número y peso (Sab 11:20).

Por eso para los Padres de la Iglesia los números pueden constituir un instrumento con el que el intelecto puede transcender de lo terrestre a lo celeste ${ }^{12}$. En los números san Agustín veía una imagen de lo absoluto, y es a través de las formas visibles como lo invisible se manifiesta a los hombres ${ }^{13}$, y en consecuencia utilizó el círculo (tras descartar el triángulo y el cuadrado) como la forma geométrica más adecuada para representar la unidad del alma, por ser una superficie limitada exclusivamente por una única línea indivisible ${ }^{14}$. Y Casiodoro, en un pasaje que debió de calar hondo en la conciencia de su tiempo, pues fue reutilizado en el siglo IX por Rabano Mauro ${ }^{15}$, especifica que:

Nunc ad geometricam veniamus, quae est descriptio contemplativa formarum, documentum etiam visibile philosophorum; quod ut preconiis efferant, Jovem suum in operibus propriis geometrizare testantur. Quod nescio utrum laudibus an vituperationibus applicetur, quando quod illi pingunt in pulvere colorato, Jovem facere mentiuntur in caelo. Quod si vero Creatori et omnipotenti Domino salubriter applicetur, potest haec sententia forsitan convenire veritati - geometrizat enim, si fas est dicere, sancta Trinitas, quando creaturis suis, quas hodieque fecit existere, diversas species formulasque concedit; quando cursus stellarum potentia veneranda distribuit, et statutis lineis facit currere quae moventur certaque sede quae sunt fixa constituit; quidquid enim bene disponitur atque completur, potest disciplinae huius qualitatibus applicari ${ }^{16}$.

11 "Número es la multitud constituida por unidades", "el uno y no el número es el origen de los números"; "la unidad no es número, pero a partir de él se siguen los números", Rabano Mauro, De Computo 3.

${ }^{12}$ SAN AGUSTíN, De ordine 15, 42-43 (Geometria et Astronomia); De civitate Dei 11, 30.

${ }^{13}$ SAN AGUSTÍN, De Trinitate 2, 5, 10.

${ }^{14}$ SAN AGUSTÍN, De quantitate animae 10-16.

${ }^{15}$ De institutione clericorum III, 23.

${ }^{16}$ Cassiodori Senatoris Institutiones, ed. Roger A.B. MYNORS, Oxford, Clarendon Press, 1937, 2.5.11, p. 150. "Pasemos ahora a la geometría, que es la descripción contemplativa de las formas, y también el modelo visual de los filósofos, porque atestiguan, según relatan en los más célebres panegíricos, que su Júpiter en sus propias obras se servía de la geometría. E ignoro si esto ha de aplicarse para alabanza o para vituperación, cuando mencionan que Júpiter hace en el cielo lo que ellos representan en arena coloreada. Porque si verdaderamente ha de aplicarse 
En algún momento entre el siglo VI y el VII Apponio en su comentario sobre el Cantar de los Cantares afirma que disciplina autem geometricae et arithmeticae artis mensura et numero omnem creaturam docet constare ${ }^{17}$.

Por eso las formas geométricas se utilizaban para construir analogías de la Divinidad. En definitiva, los números y su significado eran una de las claves para transcender con el intelecto el mundo de los sentidos, y así tratar cuestiones ontológicas recurriendo a la geometría. Especialmente las imágenes geométricas se usaban para discutir cuestiones concernientes al estatus ontológico de nociones generales (universales) en comparación con los objetos materiales. Así aparece al menos en autores tan relevantes como Macrobio, Boecio y Juan Escoto Eriúgena ${ }^{18}$.

Del mismo modo, la geometría es una herramienta para la meditación sobre la unidad metafísica (que para la religión cristiana no puede ser otra cosa sino Dios), un modo de simbolizar visualmente y contemplar la esencia incomprensible ${ }^{19}$. La esencia de la unidad inaprehensible suele ser representada por el círculo (recuérdese que tanto la longitud de la circunferencia como el área del círculo están determinados por el número irracional $P i$ ), aunque su manifestación es el cuadrado: éste, formado por dos pares perfectamente iguales aunque opuestos de ángulos, es perfectamente simétrico, comprensible (puede medirse exactamente), y representa las cuatro orientaciones primarias, los cuatro elementos (por eso se lo utiliza como expresión geométrica de la Naturaleza).

En las formas geométricas concurre además el simbolismo de los números incrementado por el hecho de que en ellas éstos interactúan estableciendo relaciones que se expresan en ratios y proporciones. La ratio es la comparación entre dos números, longitudes o magnitudes diferentes (a:b); la proporción es la relación de equivalencia entre dos ratios (a:b::c:d) y por ello representa un nivel más sutil y profundo de inteligencia que la simple percepción de la diferencia $(\text { ratio })^{20}$.

\section{Empleo de algunas superficies armónicas en la mise en page y propuesta de interpretación}

El empleo tanto de la aritmología como de las proporciones armónicas de las figuras geométricas es bien conocido en el diseño de catedrales y otros edificios,

salvíficamente a Dios creador y omnipotente, acaso francamente puede convenir a la verdad. Pues hace geometría la divinidad - si se me permite expresarlo así - cuando concede figuras y composiciones a su criatura, a la cual incluso hasta hoy mantiene en la existencia; cuando con poder digno de admiración distribuyó los cursos de las estrellas e hizo pasar en líneas preestablecidas a las estrellas que se mueven, y designó en un lugar determinado a las que están fijas".

${ }^{17}$ APPONIUS, In Canticum Canticorum expositio, CCCM 19, Turnhout, Brepols, 1986, p. 217. Cit. Eugeny A. ZAITSEV, "The meaning of early medieval Geometry: from Euclid and Surveyors' manuals to Christian Philosophy", Isis 90/3 (1999), pp 522-553, cit. p. 538.

${ }^{18}$ ZAITSEV, "The meaning of early medieval Geometry", p. 544-545.

${ }^{19}$ Robert LAWLOR, Sacred Geometry. Philosophy, London, Thames \& Hudson, 1982 (reimpr. 2002), pp. 16 y ss.

${ }^{20}$ Lawlor, op. cit., p. 44. 
que utilizan sus connotaciones de perfección formal y misticismo numérico y las trasladan a los espacios que construyen. Por ejemplo, la capilla del palacio de Carlomagno en Aquisgrán está diseñada sobre las formas de un cuadrado y un círculo, y su sección longitudinal está estructurada sobre dos círculos de 48 pies inscritos en un círculo mayor de 96 pies.

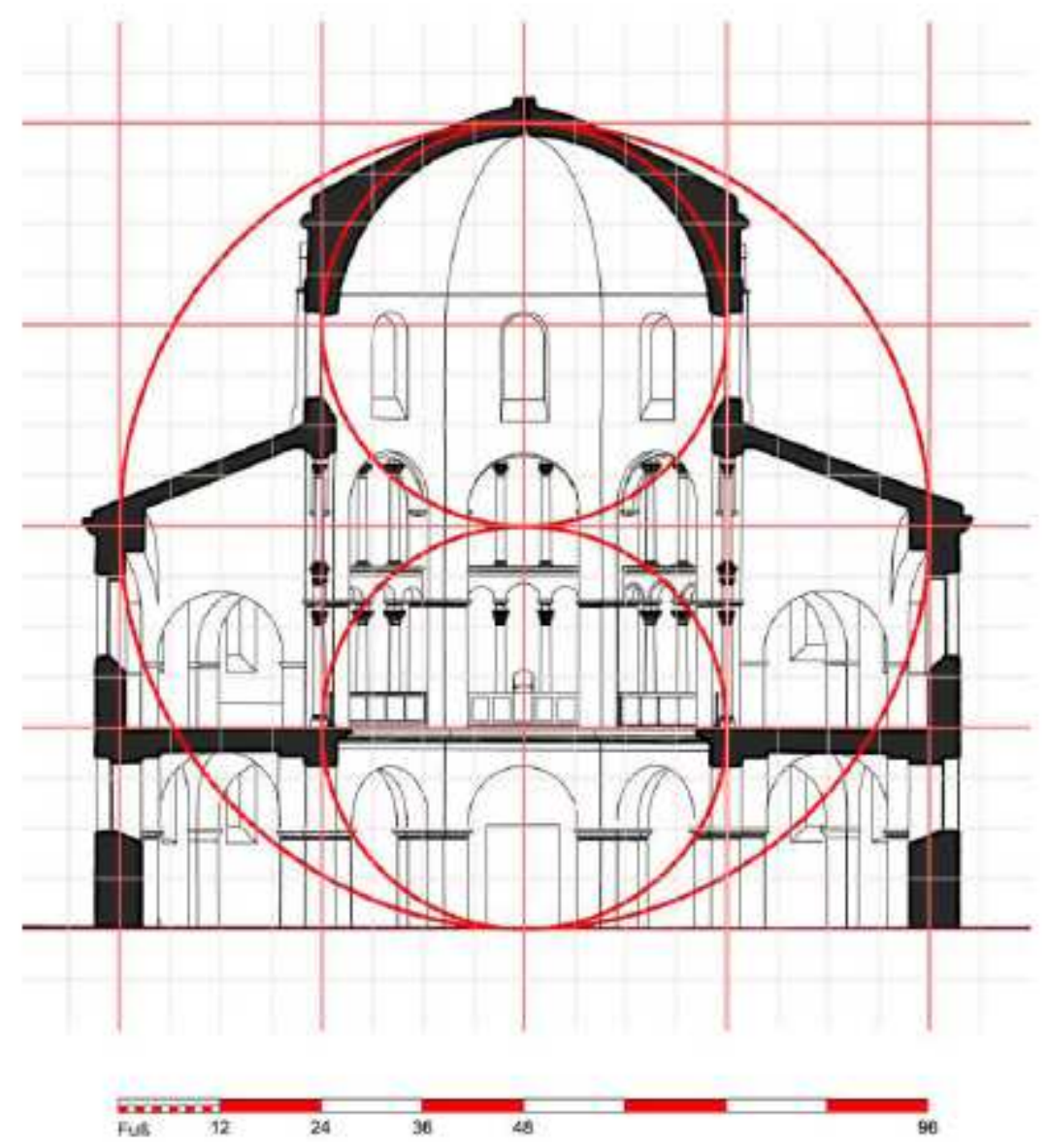

Fig. 3. "Geometría perfecta" de la capilla palatina de Aquisgrán.

(http://www.denkmalpflege.lvr.de/dokumentation/zurgeometriederpfalzkapelleaachen.pdf, [consulta 3/12/2012])

Sin embargo, en codicología el estudio de estas mismas superficies y proporciones, aunque conocido, no ha rendido los mismos resultados. Es verdad que Léon Gilissen y Jan Tschichold han identificado diversas superficies armónicas. Los estudios se han limitado en líneas generales a determinar la relación entre los dos lados del rectángulo, expresados en longitudes absolutas en el sistema métrico decimal actual, o en términos de relación entre ambas longitudes, para destacar la frecuencia con la que concurren superficies "non-arbitrary" 21 o "remarquable" 22 , en cuanto que manifiestan combinaciones de números con propiedades matemáticas curiosas, únicas o que permiten resolver problemas complejos o al menos simplificarlos. Pero ni siquiera existe un acuerdo general en

${ }^{21}$ J. TSCHICHOLD, "Non arbitrary proportions", p. 179-180.

${ }^{22}$ L. GILISSEN, Prolégomènes à la codicologie, Gand, 1977 (2a parte, "La mise en page", pp. 125-349). 
determinar qué superficies caen dentro de estas categorías, pues mientras que J. Tschichold se limita a las ratios como 1/2, 2/3, 3/4, 5/8 y 5/9 y algunas otras según aparecen en un único rectángulo, L. Gilissen considera las proporciones entre los diferentes rectángulos armónicos de su lista según aparecen combinados en la superficie de la página.

Además, aunque tanto J. Tschihold como L. Gilissen han realizado diferentes intentos de reproducir el proceso por el que se diseñaban las superficies armónicas de la página, lo cierto es que ignoramos cómo eran obtenidas en la Edad Media. Lo más probable es que, en efecto, los diseñadores gráficos de aquella época utilizaran inicialmente métodos similares (si no los mismos) a los ensayados por Tschihold y Gilissen, pero que luego se transmitirían de forma empírica, simplemente por imitación, según se deduce de la "receta" de impaginación contenida en el manuscrito parisino 11884 reproducida más arriba en este mismo artículo, aunque los más expertos podían desear experimentar con los tamaños y las proporciones. $\mathrm{Al}$ menos esto es lo que parece poder entenderse a partir de las palabras del famoso monje Teófilo, maestro de artesanos y artistas:

Per spiritum intellectus cepisti capacitatem ingenii, quo ordine qua varietate qua mensura valeas insistere diverso operi tuo ${ }^{23}$.

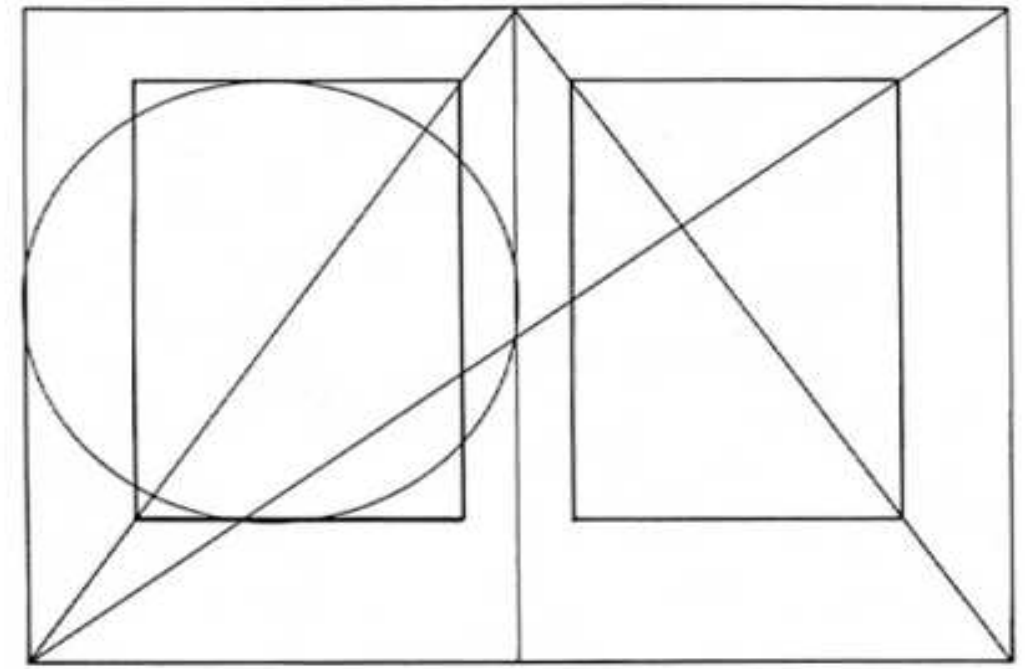

Fig. 4. Diseño del canon secreto según Tschichold. La anchura de la página es idéntica a la altura de la caja de escritura. El uso de las distintas diagonales para determinar la posición de la caja de escritura produce una perfecta integración entre ésta y la superficie de la página y la doble página. (Van de Graaf y Rosarivo consiguieron resultados idénticos utilizando métodos distintos)

${ }^{23}$ THEOPHILUS, De diversis artibus. The Various Arts. Translated from the Latin with Introduction and Notes by C.R. DODWEL, London, Nelson, 1961, p. 62. "Por el espíritu de la inteligencia (el don de la inteligencia es uno de los siete dones del Espíritu) recibiste la capacidad de crear, en qué orden, en qué diversidad, en qué medida quieres aplicar cada una de tus obras". 


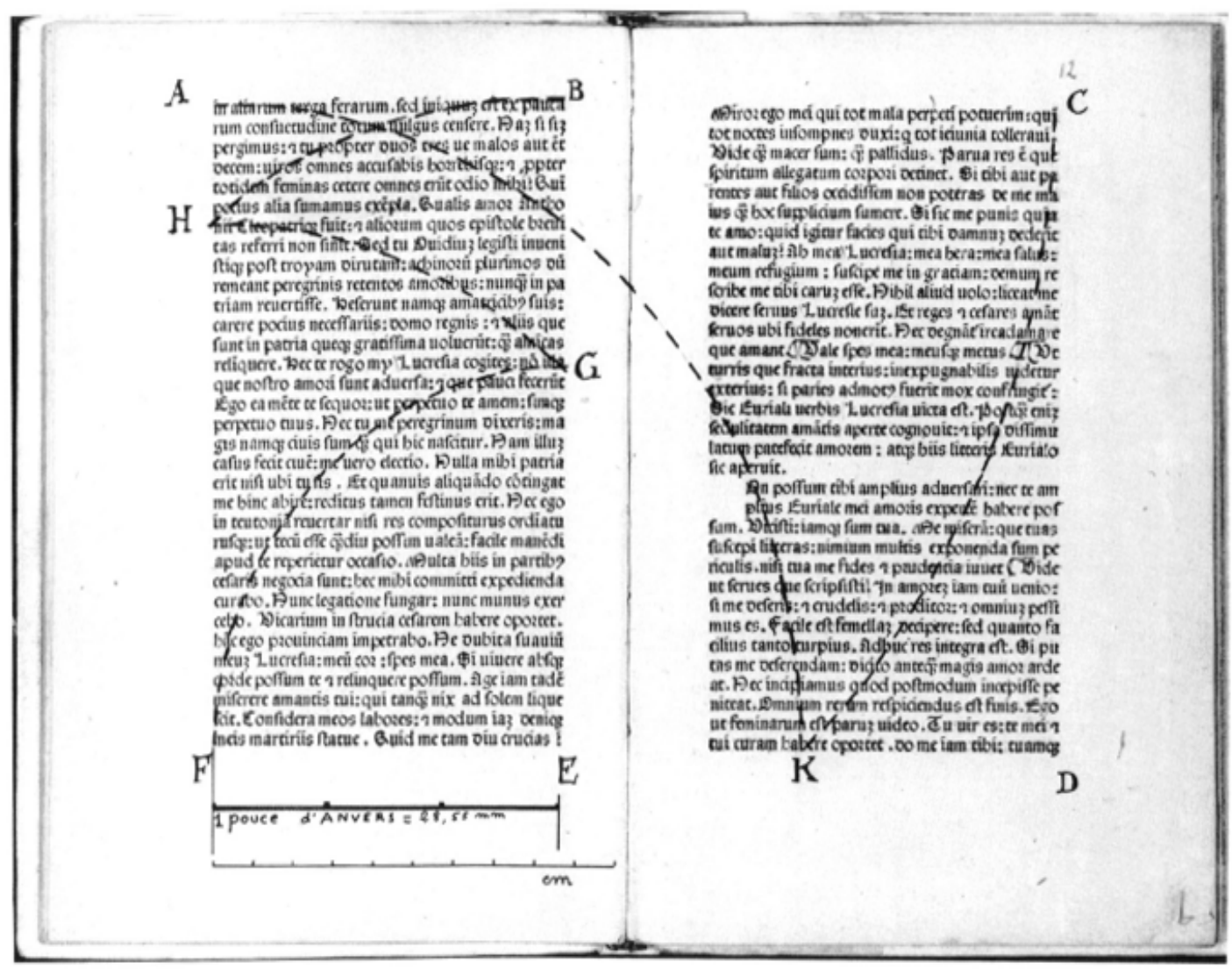

Fig. 5. Bruxelles, B.R. Inc. A 1328, Aeneas Silvius Piccolomini, De duobus amantibus, 1473

(Tomado de L. Gilissen, Prolégomènes a la Codicologie, p. 150).

Tanto la doble página como el rectángulo formado por la doble caja de escritura son sendos rectángulos de proporción creciente $1: \sqrt{2}$. La caja de escritura $1: \sqrt{ } 3$.

Como se aprecia, es muy posible que el diseño original de las superficies armónicas se hiciera simplemente con un compás, sin necesidad de tomar medidas de longitud.

Ahora bien, cualquier afirmación en este sentido no puede ser más que una suposición, ya que en la inmensa mayoría de los catálogos de manuscritos solo se expresan las dimensiones de la página actual (que ha sido probablemente alterada por algún encuadernador posterior tratando de igualar los cantos del libro), más raramente las de la caja de escritura, y prácticamente nunca la posición de esta dentro de aquella. Ello implica que cualquier estudio en esta dirección tendrá que basarse en conjuntos de manuscritos muy limitados, accesibles al investigador de un modo u otro, en lugar de utilizar grandes bases de datos que además de proporcionar resultados globales estadísticamente expresados permitirían establecer una distribución de estos en el espacio y en el tiempo.

Otra dimensión en la que existe un gran hueco en la investigación en el estudio de las superficies armónicas y su empleo en los códices es la semiótica. A menudo se ha alegado que desde el punto de vista estético las propiedades algebraicas de los rectángulos obtenidos contribuyen a imprimir en la página una impresión de armonía que es agradable al ojo. Pero lo cierto es que los números eran entendidos como signos de realidades espirituales y las figuras geométricas jugaban un papel 
fundamental en el simbolismo de las edificaciones religiosas ${ }^{24}$, e igual que en los constructores de catedrales, existió sin duda entre los mejores maestros de escriptorio una intencionalidad de transcender la mera estética y proporcionar a los lectores de sus libros una herramienta de meditación a través de la simple contemplación de las formas geométricas.

Ya se ha comentado más arriba que según la definición de Boecio (tomada de Nicómaco de Gerasa) proporción es la relación de equivalencia entre dos ratios y que ratio es a su vez la comparación entre dos números. Es decir, que en principio dos ratios implican cuatro números diferentes (a:b::c:d; ej.: 2:4::3:6), relacionados a un nivel que en el pensamiento griego se define como analogía ${ }^{25}$, y recuérdese que cuatro es precisamente el número simbólico para expresar la naturaleza finita, racional y mensurable del mundo creado: en él se hallan las claves de toda la naturaleza ${ }^{26}$. En la matemática pitagórica, una proporción de este tipo recibe el calificativo de "proporción discontinua".

Ahora bien, la contemplación de una proporción puede ayudar a la mente a trascender el mundo creado, cuando en la proporción los elementos implicados han sido reducidos de cuatro a tres, por medio de establecer la identidad del segundo término con el tercero: a:b::b:c (o a:b:c). Los griegos denominaron a este tipo de proporción de tres términos "proporción continua" y recuérdese que en la matemática pitagórica el tres es el número en acción, el primer número primo después de la mónada y de la díada, y se asocia a todo lo que implica "composición" en la naturaleza, y por ello conocimiento ${ }^{27}$. Pero en el mundo cristiano el tres evocaba sin duda la Trinidad...

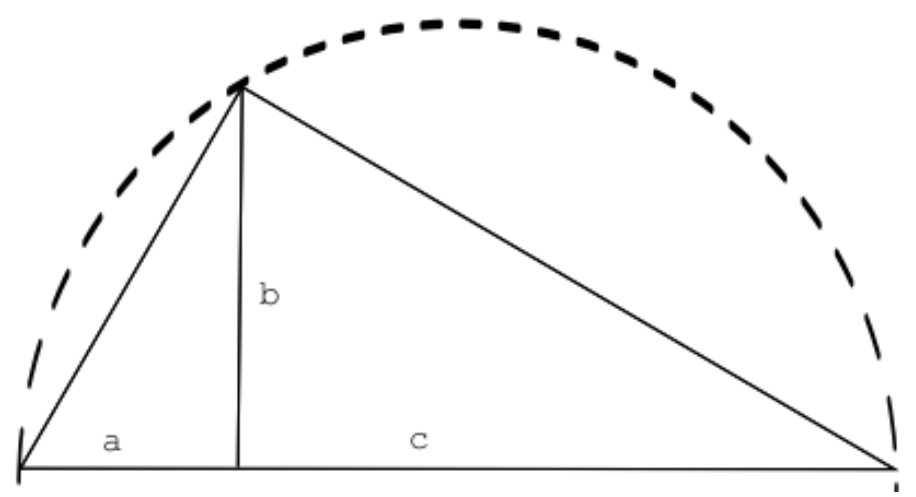

Fig. 6: Representación geométrica de una proporción continua de tres términos utilizando el teorema de Tales (Tomado de Lawlor, op. cit., p. 45).

En virtud del teorema de Tales, todo triángulo inscrito en un semicírculo es un triángulo recto. La línea perpendicular al diámetro (b) es el término medio geométrico entre los dos segmentos del diámetro $(\mathrm{a}, \mathrm{c})$ : a:b::b:c.

${ }^{24}$ Marie-Pierre TERRIEN, "Religious architecture and Mathematics during the Late Antiquity", in T. KOETSIER \& L. BERGMANS (eds.), Mathematics and the Divine: a Historical Study, Amsterdam, Elsevier, 2005, pp. 147-160.

${ }^{25}$ LAWLOR, op. cit., p. 44.

${ }^{26}$ J.F. MATTÉI, "Nicomachus of Gerasa and the Arithmetic Scale of the Divine", in T. KOETSIER \& L. BERGMANS (eds.), Mathematics and the Divine: a Historical Study, Amsterdam, Elsevier, 2005, pp. 123-132, cit. p. 128.

${ }^{27}$ MATTÉI, op cit., p. 128. 
Entre las superficies armónicas identificadas por Gilissen y Tschichold una de las más asiduamente utilizadas en los manuscritos medievales es la llamada proporción áurea, que se define por el número irracional Phi $(1,618 \ldots)$. Phi puede definirse aritméticamente como el número cuyo cuadrado se obtiene sumando 1 al número inicial $\left(P h i^{2}=P h i+1\right)$, de donde $P h i=(\sqrt{ } 5+1) / 2^{28}$. Pero Phi tiene también una definición geométrica que para nuestro propósito es todavía más interesante. $P h i$ o proporción áurea tiene la peculiaridad de que es la única proporción que puede definirse con solo dos términos: a:b::b:(a+b), es decir, que la ratio entre $a$ y $b$ es idéntica a la ratio entre $b$ y el conjunto del total. Tres que proceden de dos y que en realidad son uno... geométricamente no se puede estar más cerca del misterio teológico de la Santísima Trinidad, y misterio también geométrico, porque Phi es un número irracional, que no se puede medir, aunque se pueda representar, y que es expresión de la proporción sobre la que se funda la experiencia del conocimiento o logos, y Logos (=Lat. Verbum) es también la expresión de Dios-Hijo: In principio erat Verbum, et Verbum erat apud Deum, et Deus erat Verbum (Io 1:1).

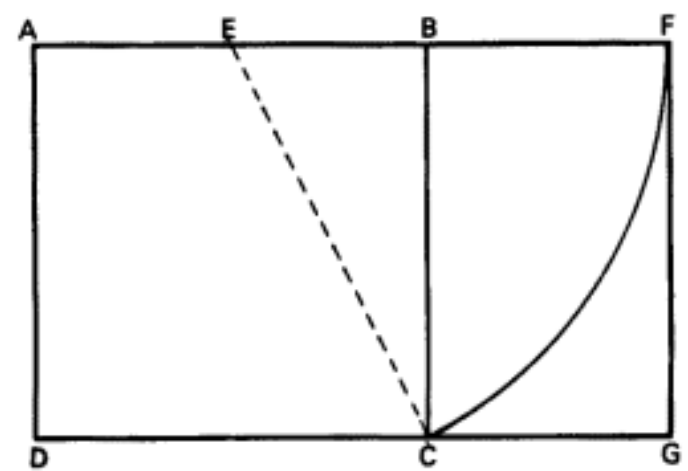

Fig. 7. Construcción geométrica de un rectángulo áureo.

(Tomado de H. E. Huntley, The Divine Proportion. A Study of Mathematical Beauty, New York, Dover Publications, 1970, p. 61).

La historia del arte religioso proporciona abundantes ejemplos del empleo de Phi como medio para alcanzar balance en el diseño compositivo. Según Frederik Macody Lund, numerosas estructuras arquitectónicas góticas están diseñadas siguiendo la dorada proporción, entre otras las catedrales de Chartres, Laon y Notre-Dame de París, pero también las esbeltas proporciones de las estatuas de estas mismas catedrales se corresponden con ella ${ }^{29}$.

En el terreno codicológico Robert Stevick ha examinado algunas páginas iluminadas de manuscritos, con el propósito de descubrir las técnicas de diseño de sus autores. Su conclusión es que la dorada proporción es la piedra angular del diseño, y que la impaginación está planificada utilizando instrumentos similares, aunque de menor tamaño, a los usados por los constructores de catedrales ${ }^{30}$.

${ }^{27} \mathrm{O}(\sqrt{ } 5-1) / 2$.

${ }^{29}$ James SNYDER, Medieval Art: Painting, Sculpture, Architecture, 4th-14th Century, Upper Saddle River, N.J, Prentice Hall, 1989, p. 260.

${ }^{30}$ Robert D. STEVICK, “The Echternach Gospels' Evangelist Symbol Pages: Forms from 'The Two True Measures of Geometry”", Peritia 5 (1986), pp. 284-308. 


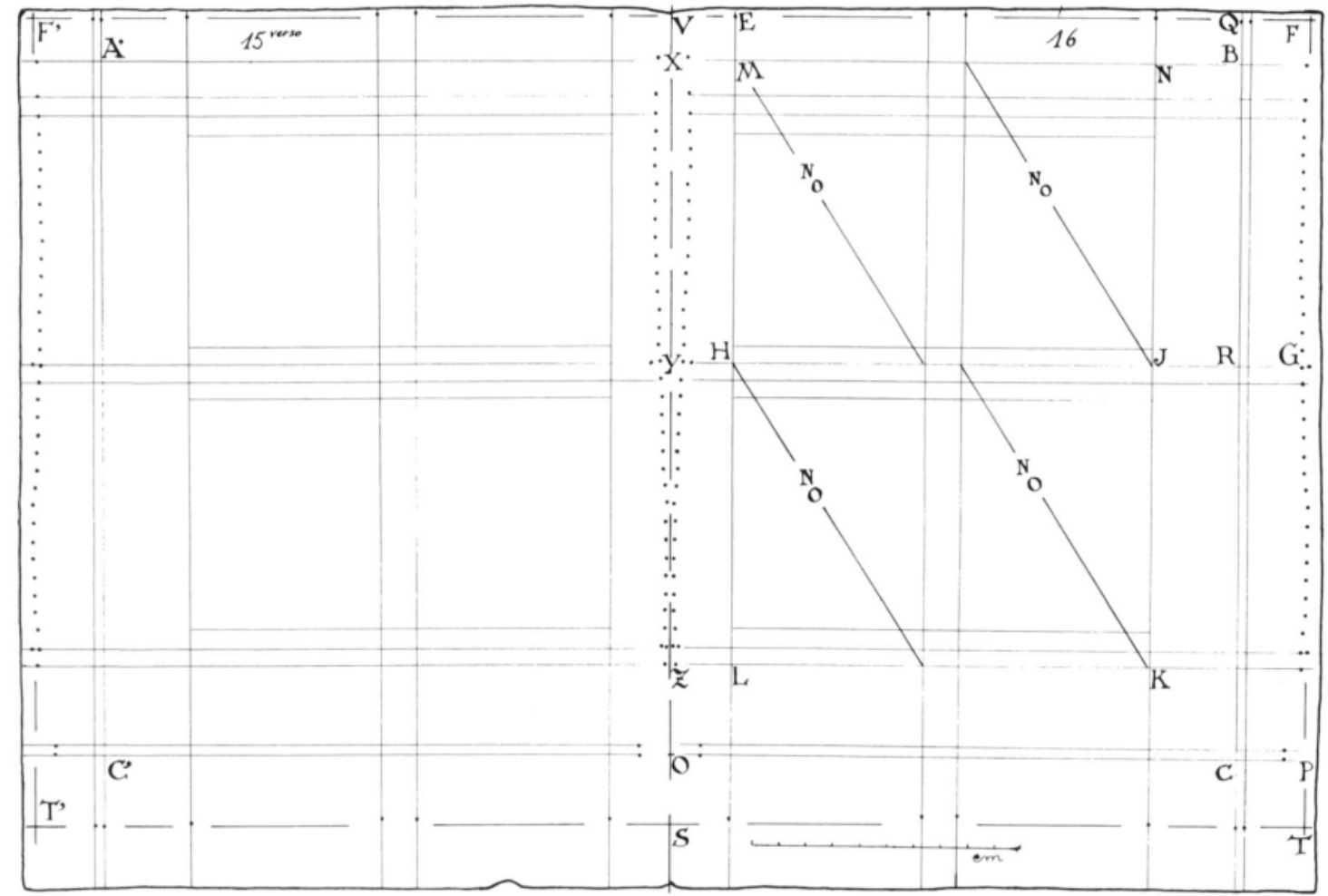

Fig. 8. La dorada proporción como elemento articulador del diseño de la página en Bruselas, B.R., II 1108, S.Agustín, De civitate Dei (siglo XIV).

(Tomado de Gilissen, Prolégomènes..., fig. 57, pp. 206-207).

Finalmente, otra de las superficies armónicas identificadas en los códices es el rectángulo de proporción creciente. Se define por tener su lado mayor la longitud de la diagonal de un cuadrado formado a partir de su lado menor (proporción $1: \sqrt{2}$ ). La matemática de esta figura también se remonta a Grecia, donde se conoció el fenómeno como "expansión gnómica". Según Herón de Alejandría un gnomon es una figura que añadida a la figura original produce una figura similar a la figura original, y la contemplación de esta figura conduce a la comprensión de una de las formas más conocidas de crecimiento natural: el crecimiento por acreción o incremento acumulativo, en el cual la antigua forma está contenida dentro de la nueva $^{31}$. Nos encontramos aquí frente a un proceso de expansión indefinida en el que cada uno de los términos contiene, superados, a cada uno de los que le han precedido y preludia a su vez el paso siguiente. No en vano este es el principio que sigue el actual formato DIN, en el cual cada tamaño produce exactamente el tamaño siguiente al ser doblado por la mitad de su lado mayor. Pero además el empleo de $\sqrt{ } 2$ le confiere un significado especial, al menos a juzgar por la práctica equivalente en la arquitectura religiosa, donde los maestros constructores vieron una especie de término medio entre 1 y 2 , considerados como dos extremos opuestos (lo cual es precisamente la definición aristotélica de virtud). 1: $\sqrt{2}: 2$ es exactamente la proporción geométrica según es descrita por Boecio, y en los claustros define la relación entre el cuadrado interior y el exterior, de modo que caminar por él podía ser interpretado como recorrer el camino de la virtud que

31 LAWLOR, op. cit., p. 65. 
conduce al Reino de los Cielos $^{32}$. En un códice, semejante proporción estaría representada por tres rectángulos de proporción creciente $1: \sqrt{ } 2$, cuya ratio entre áreas es 1:2:4, dispuestos de la manera que se indica en la Fig. 9.

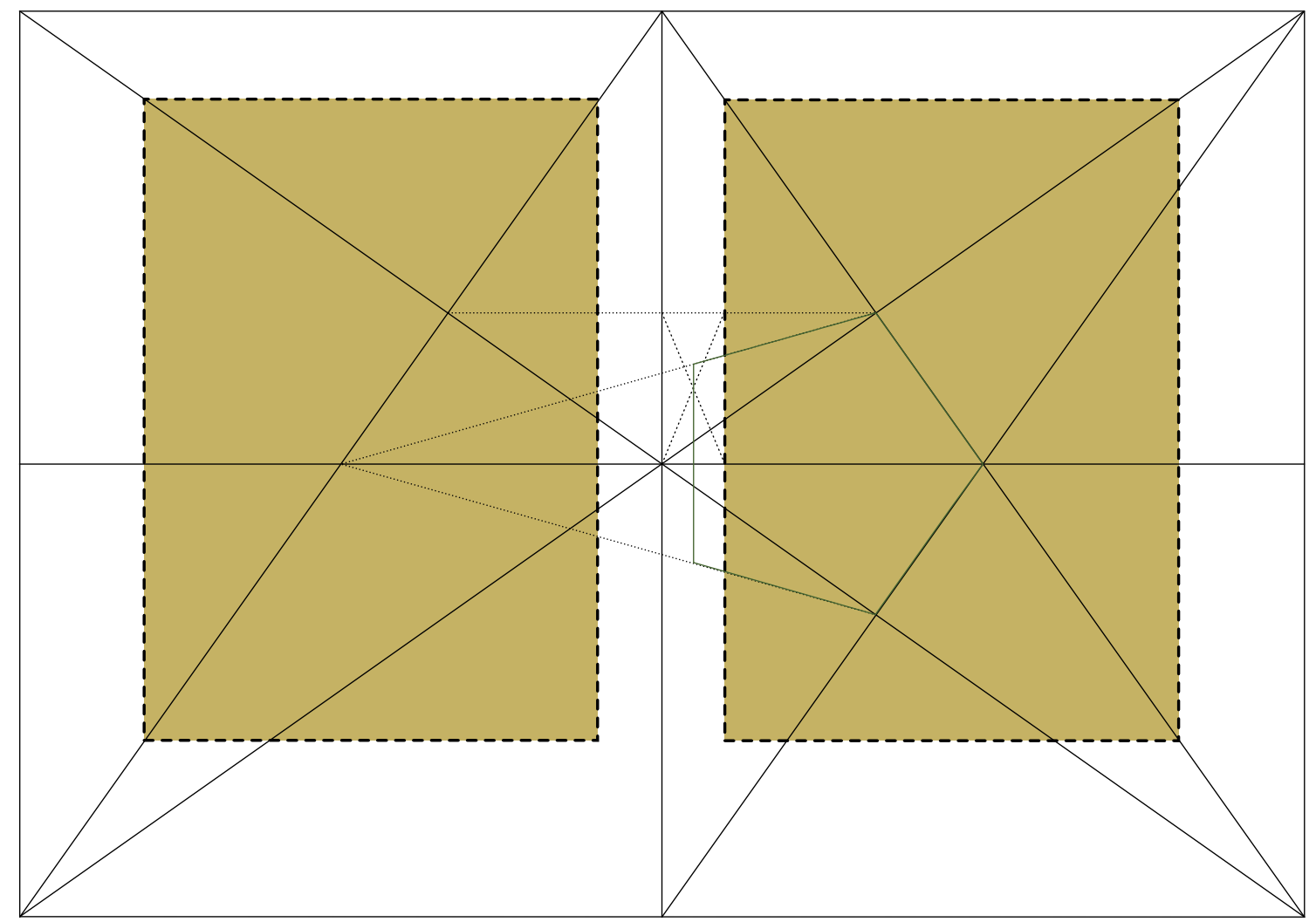

Figura 9: Tres rectángulos de proporción creciente: La doble página, cada una de las páginas y las cajas de escritura, cada una respectivamente la mitad que la anterior. La base del pentágono regular divide en dos el margen interno, de modo que la proporción de las anchuras de los márgenes es 2:3:4:6, que forman además la proporción armónica de Boecio.

El rectángulo de proporción creciente en proporción 1: $\sqrt{2}$ es uno de los más utilizados en los códices medievales. Utilizando la diagonal de este rectángulo para construir uno nuevo, se obtiene una proporción de $1: \sqrt{3}$, y la progresión puede continuarse indefinidamente $(1: \sqrt{ } 4,1: \sqrt{ } 5 \ldots)$.

Entre los diseños de página estudiados por Léon Gilissen, los rectángulos de proporción creciente $1: \sqrt{2}$ y $1: \sqrt{ } 3$ se hallan entre los más numerosos, y se da el caso además de que esta proporción es la más abundante en los manuscritos latinos de los siglos XIII y XIV de la Biblioteca Histórica Marqués de Valdecilla de la Universidad Complutense de Madrid ${ }^{33}$.

32 David CLAYTON, "Harmonious Proportion and Ratio", Second Spring. A Journal of Faith and Culture, (2009) [http://www.secondspring.co.uk/articles/Clayton_HarmoniousProportion-and-Ratio.pdf, consulta 1/12/2012] cit. p. 5.

${ }^{33}$ En 2009 tuve la ocasión de dirigir el Trabajo de Fin de Máster de José María Fernández Ramón en el que se analizaba el diseño de la página en una muestra de códices hebreos y latinos conservados en la Biblioteca Histórica "Marqués de Valdecilla" de la Universidad Complutense; en la muestra estudiada el rectángulo proporcional $1: \sqrt{ } 2$ se halla entre las proporciones más utilizadas en los códices latinos, solo superada por el rectángulo de Pitágoras $(3: 4)$. 


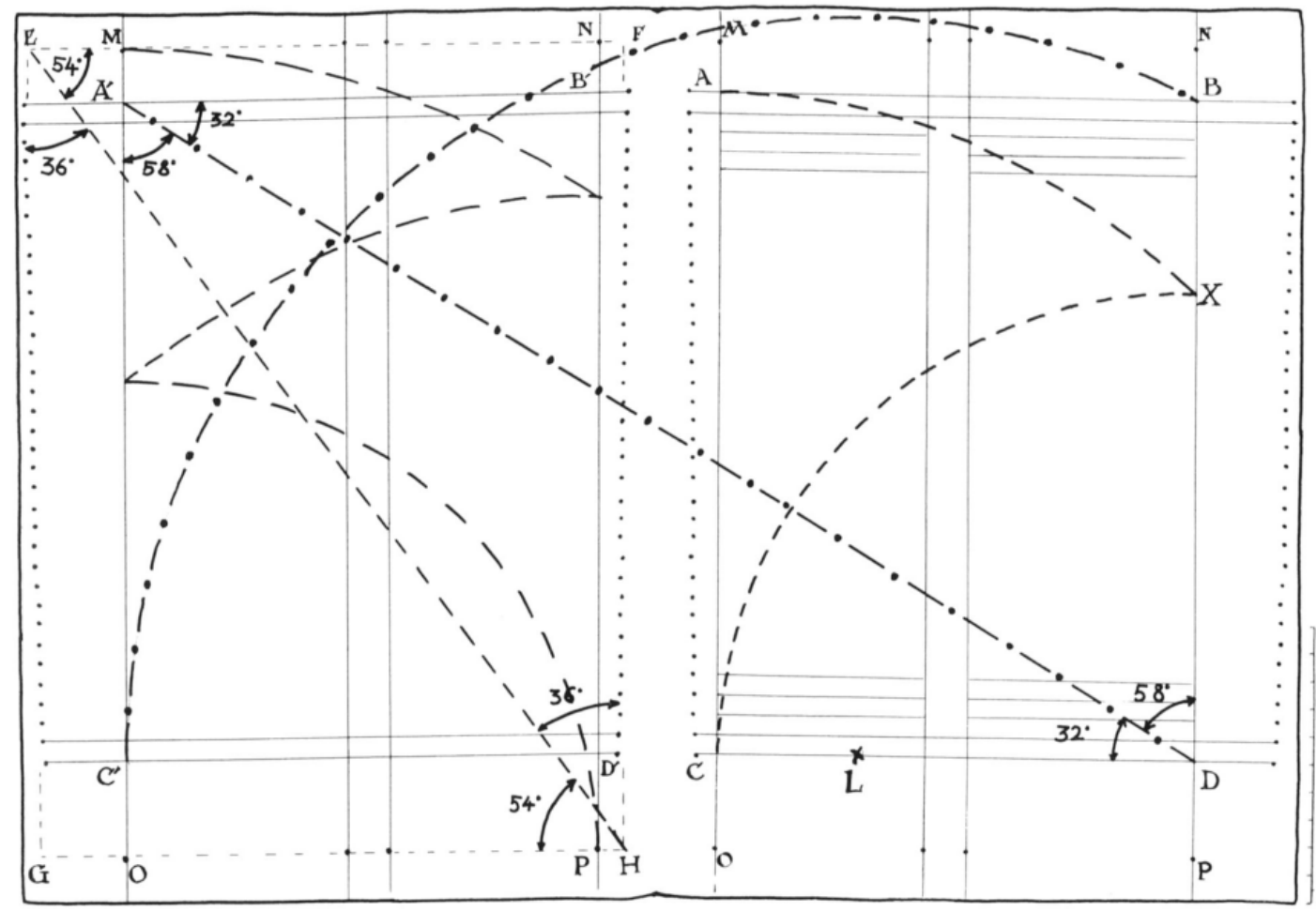

Fig. 10. Ms. Bruselas, B.R. II 1106 (Tomado de L. Gilissen, Prolégomènes..., p. 140-141).

La doble página, cada una de las páginas y la caja de escritura son rectángulos proporcionales 1: $\sqrt{2}$.

Para la ubicación de la caja de escritura sobre la página se ha utilizado un rectángulo proporcional 1: $\sqrt{3}$.

\section{Interpretación de los valores absolutos de los números}

Otra cuestión relativa a la mise en page susceptible de ser estudiada desde un punto de vista semiótico es la de las medidas absolutas. Un tanto de pasada ya se ha mencionado la cuestión del valor simbólico de ciertos números. Y, en efecto, la numerología o misticismo numérico fue una disciplina asiduamente cultivada en la Edad Media. Por ejemplo, fueron cultivadores entusiastas de esta modalidad de las matemáticas Marciano Capella (De nuptiis, II, 7), san Isidoro (Liber numerorum) y Rabano Mauro (De numero). Isidoro y Rabano recogen los atributos pitagóricos de cada número, pero añadiendo aplicaciones teológicas, tomadas tanto de la Biblia como de los Padres. Isidoro alcanza a comentar hasta el 153, pero Rabano ( $D e$ numero) extiende la lista hasta el 144.000, esto es, al número final de los elegidos, $\mathrm{y}$, aunque el Sevillano dé mayor número de ejemplos, Rabano es más refinado en su elaboración matemática ${ }^{34}$.

Cabe suponer, por tanto, que las medidas absolutas de la página, la caja de escritura o los márgenes no carecieran de valor simbólico. La dificultad para proseguir una investigación en esta dirección deriva de la extrema falta de regulación de las unidades de medida durante la Edad Media, ya que por supuesto no existía el sistema métrico decimal, y todo lo relativo a pesos y medidas se atenía

34 V.F. HOPPER, Medieval Number Symbolism. Its Sources, Meaning and Influence on Thought and Expression, New York, Columbia University Press, 1938, p. 104. Sobre el misticismo numérico específicamente en Rabano Mauro ver B. TAEGER, Zahlensymbolik bei Hraban, bei Hincmar und im 'Heliand'. Studien zur Zahlensymbolik im Frühmittelalter. München, C.H. Beck’sche Verlagsbuchhandlung, 1970. 
a costumbres locales y difería considerablemente de unos lugares a otros ${ }^{35}$. Por ejemplo, en la Cataluña altomedieval la unidad de medida básica parece haber sido el pie, pero es difícil asegurar su correspondencia en el actual sistema métrico decimal, porque se usaban diversos tipos de pies: pie carolingio $(33,048 \mathrm{~cm})$, pie romano $(29,376 \mathrm{~cm})$, y otros dos pies catalanes más (de 29,156 y 25,930 cm respectivamente $)^{36}$. Y al menos al final de la Edad Media y principios de la Edad

${ }^{35}$ Aquí se incluye alguna bibliografía sobre la cuestión, separada por áreas geográficas:

General: Antonio TORRENTS Y MONNER, Tratado de monedas, pesas y medidas antiguas y modernas de todos los países, Barcelona, Bayer Hnos, 1915. Paul BURGUBURU, Essai de bibliographie métrologique universelle, Paris, Auguste PICARD, 1932. Witold KULA, Las medidas y los hombres, Madrid, Siglo XXI, 1980. Bernard GARNIER, Jean-Claude HOCQUET \& Denis WORONOFF, Introduction a la métrologie historique, Paris, Economica, 1989. Alex HEBRA, Measure for measure: the story of imperial, metric and other units, Baltimore, The John Hopkins University Press, 2003. Andrew ROBINSON, Metrum: historia de las medidas, Barcelona, Paidós, 2007. PELLICER I BRU, Josep, Metrología. Diccionario descriptivo, Barcelona, Asociación numismática española, 2011.

Península Ibérica: a) General: Ana Ma ROMÁN GARCÍA, "Notas para una bibliografía metrológica española", Boletín de Metrología 1 (1954), pp. 78-97; 2 (1955), pp. 59-76; 3 (1956), pp. 49-70. b) Corona de Castilla: Ordenanzas reales de Castilla, lib. V, tit. VII. Novísima recopilación de las leyes de España, Madrid, Rivadeneyra, 1850, lib. IX, tit. IX. Ignacio CARRIÓN ARREGUI, "Los antiguos pesos y medidas guipuzcoanos", Vasconia 24 (1996), pp. 59-79. J. COBO AVILA, "Estudio preliminar", en A.M. BURRIEL, Informe de la Ciudad de Toledo al Consejo de Castilla sobre igualación de pesos y medidas [1758], Toledo, Diputación, 1991, pp. 7-43. c) Corona de Aragón: Pablo LARA IZQUIERDO, Sistema aragonés de pesos y medidas: la metrología histórica aragonesa y sus relaciones con la castellana, Zaragoza, Guara, 1984. Gaspar, FELIÚ I MONTFORT, "Algunes consideracions sobre la metrologia altmedieval catalana", Acta historica et archaeologica mediaevalia 22 (2001), pp. 121-149.

Italia: R.E. ZUPKO, Italian Weights and Measures from the Middle Ages to the Nineteenth Century, Philadelphia, The American Philosophical Society, 1981.

Francia: Pierre CHARBONNIER (dir.), Les anciennes mesures du Centre-Est d'après les Tables de conversion, Coll. Histoires Croisées, CHEC de Clermont-Ferrand, Presses Universitaires Blaise Pascal, 2005. Jean-Claude HOCQUET, "La métrologie, voi nouvelle de la recherche historique", Comptes rendus de l'Académie des Inscriptions et Belles-Lettres, Paris, 1990, pp. 59-76. Pierre PORTET, "Remarques sur la métrologie carolingienne", Le Moyen-Age, 5e série 1 (1991), pp. 5-24.

Países Bajos: Auguste VINCENT, "Notes de Métrologie. Les mesures locales dans les incunables", Revue Belge de Philologie et d'Histoire 5 (1926), pp. 955-966.

Alemania: Harald WITTHÖFT, Umrisee einer historischen Metrologie zum Nutzen der wirtschafts-und sozialgeschichtlichen Forschung: Mass und Gewicht in Stadt und Land Lüneburg, in Hansenraum und im Kurfürstentum/Königreich Hannover von 13. bis zum 19. Jahrhundert, Veröffentlichungen des Max-Plancks-Instituts für Geschichte 60/1, Göttingen, Vandenhoek \& Ruprecht, 1979.

Islas Británicas: F.G. SKINNER, Weights and Measures: their Ancient Origins, London, Her Majesty's Stationery Office, 1967. Ronald Edward Zupko, British Weights and Measures: A History from Antiquity to the Seventeenth Century, Madison, Wi., University of Wisconsin Press, 1978.

36 Gaspar FELIÚ I MONFORT, "Algunes consideracions sobre la metrologia altmedieval catalana", pp. 140-141. 
Moderna, en Castilla el patrón de medida lineal era la vara de Burgos, de 0,835905 metros, con pequeñísimas diferencias locales, debidas con toda probabilidad a la dificultad técnica para realizar medidas exactas, y la vara se dividía a su vez en dos codos; la vara se dividía en codos (2/3), pies (1/3), palmos (1/3 ó 1/12), pulgadas (1/36), dedos (1/48); una pulgada tenía 12 líneas y la línea 12 puntos ${ }^{37}$. Los ejemplos similares podrían multiplicarse para prácticamente la totalidad de las regiones europeas.

O sea, que intentar convertir las medidas de una página medieval desde el sistema métrico decimal actual a su sistema métrico originario implicaría para empezar datar temporal y especialmente el manuscrito y en segundo lugar tantear las distintas posibilidades que podría ofrecer el sistema métrico local ${ }^{38}$, para solo entonces poder comenzar a especular con el significado simbólico implicado en los números. Y por supuesto siempre quedaría la posibilidad de que los maestros de escriptorio utilizasen fórmulas aritméticas con instrumentos no métricos, como reglas plegables o cuerdas anudadas a ciertos intervalos. Por estas y otras razones, cualesquier conclusiones a las que se pudiera llegar partiendo de las unidades métricas no pueden ser más que provisionales.

\section{Conclusiones}

En conclusión, la confección de un manuscrito requería muchas más operaciones que simplemente la preparación del material y la copia del texto. Había muchas decisiones que tomar y muchas opciones para elegir en cada una de ellas.

Igual que en lo relativo a la elección de la escritura, donde el maestro de escriptorio podía jugar con los tipos de letra no solo para dar mayor o menor importancia a ciertas partes del texto, sino también para provocar en el lector ciertas impresiones; en lo que se refiere al diseño de la doble página, tenía ante sí un muestrario de elementos que tenía que combinar para conseguir un resultado equilibrado y estéticamente satisfactorio. $\mathrm{Y}$ aunque en muchas veces estas elecciones se produjeran de forma más o menos rutinaria o incluso aplicando plantillas ya diseñadas con antelación o copiando de otros libros, en otras sin duda se buscaría crear nuevas combinaciones.

Y es muy probable que en este último caso el diseño de la doble página siguiera pautas similares a las que se han detectado en el diseño de las plantas y alzados de catedrales y otros edificios, donde se juega con el valor simbólico de los números y sus combinaciones. El número áureo, el rectángulo de proporción creciente y otras superficies armónicas similares, más allá de la sensación de equilibrio y armonía que producen en el lector actual de la página, podían ser interpretados durante la Edad Media como expresiones de realidades metafísicas.

Pero en el estado actual de los estudios codicológicos es imposible sacar conclusiones generales en este sentido, ya que carecemos de series de datos

37 CARRIÓN ARREGUI, op. cit., p. 62.

38 Acaso pudiera utilizarse un método similar al empleado por Nicola MASINI, Cosimo Damiano FONSECA, Edoardo GERALDI y Gabriella SABINO, "An algorithm for cumputing the original units of measure of medieval architecture", Journal of Cultural Heritage 5 (2004), pp. 7-15. 
suficientemente amplias para ser relevantes, debido a que en su inmensa mayoría los catálogos de bibliotecas solo indican las medidas de la página completa, e incluso sin prestar atención si esta es la original o si ha sido recortada durante una reencuadernación.

Es necesario por tanto realizar estudios seriados enfocados en el análisis de la mise en page, sus medidas según la metrología del lugar y tiempo en que fue creada, sus distintas superficies armónicas y el modo en que estas se combinan. Cuestiones como qué tipo de superficies armónicas tienden a utilizarse en qué tipo de libros; si hay relación entre el tipo de letra y las superficies armónicas; si se percibe una evolución en el empleo de estas a través del tiempo, o si la tendencia es idéntica o no en los códices latinos, vernáculos, hebreos y árabes, son solo algunas de las direcciones que podrían tomarse a este respecto.

Pero también es necesario profundizar en los valores simbólicos de estas mismas superficies armónicas, y estos solo se hallarán comparando las proporciones de los códices con las de las catedrales y otros edificios religiosos y exprimiendo los textos que puedan arrojar luz sobre la teoría de la armonía (Boecio, principalmente) y el misticismo numérico (Isidoro de Sevilla y Rabano Mauro, entre otros).

\section{Fuentes y Bibliografía}

\section{Fuentes}

Agustín (San), De civitate Dei. Texto latino y traducción española por Santos Santamarta del Río y Miguel Fuertes Lanero, Obras completas de san Agustín 16 y 18, Madrid, Editorial Católica, 1988.

Agustín (San), De ordine. Texto latino y traducción española por Victorino Capanaga, Obras completas de san Agustín 1. Escritos filosóficos, Madrid, Biblioteca de Autores Cristianos, 1994.

Agustín (San), De quantitate animae. Texto latino y traducción española por Victorino Capanaga, Obras completas de san Agustín 3. Obras filosóficas, Madrid, Editorial Católica, 1963.

Agustín (San), De Trinitate. Texto latino y traducción española por Luis Arias, Obras Completas de san Agustín 5, Madrid, Editorial Católica, 1948.

APONIO, In Canticum Canticorum expositio, CCCM 19, Turnhout, Brepols, 1986.

BoEciO, De institutione arithmetica libri duo, Lipsiae, B.G. Teubneri, 1867. Traducción española de María Asunción Sánchez Manzano, León, Universidad de León, 2002.

CASIODORO, Institutiones. Ed. Roger A.B. Mynors, Oxford, Clarendon Press, 1937.

Novísima recopilación de las leyes de España, Madrid, Rivadeneyra, 1850.

Ordenanzas Reales de Castilla, recopiladas por Alfonso Díaz de Montalvo. Existen multitud de ediciones. Texto accesible on-line: http://books.google.nl/ books?id=ILYWAAAAQAAJ\&printsec=frontcover\&hl=es\&source=gbs_ge su mmary $\mathrm{r} \& \mathrm{cad}=0$

RABAno Mauro, De computo. Ed. J. McCulloh \& W. Stevens, CCCM 44, Turnhout, Brepols, 1997. 
RABANO MAURO, De institutione clericorum. Texto latino y traducción española por Ana Belén Sánchez Prieto, Madrid, Biblioteca de Autores Cristianos, en prensa.

TEÓFILO, De diversis artibus. Texto y traducción al inglés, con notas, por C.R. Dodwel, London, Nelson, 1961.

\section{Bibliografia}

BISCHOFF, F.M. \& MANIACI, M., "Pergamentgröße, Handschriftenformate, Lagenkonstruktion. Anmerkungen zur Methodik und zu den Ergebnissen der jüngeren kodikologischen Forschung”, Scrittura e civiltà 19 (1995), pp. 277-319.

Bozzolo, Carla; CoQ, Dominique; MuZERElle, Denis \& ORnato, Ezio, "Noir et blanc. Premiers résultats d'une enquête sur la mise en page dans le livre médiéval", en Cesare Questa \& Renato Raffaelli (eds.), Atti del Convegno internazionale Il libro e il testo (Urbino, 20-23 settembre 1982), Urbino, Università degli Studi di Urbino, s.f., pp. 195-221. Reeditado en Ezio Ornato, La face cachée du livre médiéval. L'histoire du livre vue par Ezio Ornato, ses amis et ses collègues, Roma, Viella, 1997, pp. 473-508.

Bozzolo, Carla; CoQ, Dominique; Muzerelle, Denis \& Ornato, Ezio, "Page savante, page vulgaire: Étude comparative de la mise en page des livres en latin et en français écrits ou imprimés en France au XVe siècle", en É. Baumgartner $\&$ N. Boulestreau (eds.), La présentation du livre. Actes du Colloque de ParisXNanterre (Nanterre, 3-5 décembre 1985), Nanterre, 1987, pp. 121-133, reed. en La face cachée du livre médiéval. L'histoire du livre vue par Ezio Ornato, ses amis et ses collègues, Roma, Viella, 1997, pp., 509-517.

Bozzolo, Carla; CoQ, Dominique; Muzerelle, Denis \& Ornato, Ezio, "L'artisan médiéval et la page: peut-on décéler des prócédés géométriques de mise en page?", en X. BARRAL I ALTET (ed.), Artistes, artisans et production artistique au Moyen Âge. Actes du Colloque (Rennes, 2-6 mai 1983), Paris, Picard, 1990, vol. III, pp. 295-305. Republ. en La face cachée du livre médiéval. L'histoire du livre vue par Ezio Ornato, ses amis et ses collègues, Rome, Viella, 1997, pp. 447-456.

BÜHLER, C.F., "The Margins in Mediaeval Books", The Papers of the Bibliographical Society of America 40/1 (1946), pp. 32-42.

BuRguburu, Paul, Essai de bibliographie métrologique universelle, Paris, Auguste Picard, 1932.

CARrIóN ARREGUI, Ignacio, "Los antiguos pesos y medidas guipuzcoanos", Vasconia 24 (1996), pp. 59-79.

CHARBOnNIER, Pierre (dir.), Les anciennes measures du Centre-Est d'après les Tables de conversion, Coll. Histoires Croisées, CHEC de Clermont-Ferrand, Presses Universitaires Blaise Pascal, 2005.

Clayton, David, "Harmonious Proportion and Ratio", Second Spring. A Journal of Faith and Culture, (2009) http://www.secondspring.co.uk/articles/ Clayton_Harmonious-Proportion-and-Ratio.pdf [consulta 1/12/2012].

CoBo AvilA, J., "Estudio preliminar" a A.M. Burriel, Informe de la Ciudad de Toledo al Consejo de Castilla sobre igualación de pesos y medidas [1758], Toledo, Diputación, 1991, pp. 7-43. 
FELIU I MONTFORT, Gaspar, "Algunes consideracions sobre la metrologia altmedieval catalana", Acta historica et archaeologica mediaevalia 22 (2001), pp. 121-149.

GARNIER, Bernard; HOCQUET, Jean-Claude \& WORONOFF, Denis, Introduction a la metrologie historique, Paris, Economica, 1989.

GILlissen, Léon, Prolégomènes à la codicologie, Gand, 1977 (especialmente pp. 125-249).

GRAAF, John A. van de, "Nieuwe berekening voor de vormgeving", Tété (November 1946), pp. 95-100.

HEBRA, Alex, Measure for measure: the story of imperial, metric and other units, Baltimore, The John Hopkins University Press, 2003.

HOCQUET, Jean-Claude, "La métrologie, voie nouvelle de la recherche historique", Comptes rendus de l'Académie des Inscriptions et Belles-Lettres, Paris, 1990, pp. 59-76.

HOPPER, V.F., Medieval Number Symbolism. Its Sources, Meaning and Influence on Thought and Expression, New York, Columbia University Press, 1938.

Huntley, H.E., The Divine Proportion. A Study of Mathematical Beauty, New York, Dover Publications, 1970.

JOHN, J.J., "The Format of Manuscripts in 'Codices Latini Antiquiores': A Statitstical Report", Actas del VIII Coloquio del Comité Internacional de Paleografía Latina, Madrid, 1990, pp. 95-105.

KULA, Witold, Las medidas y los hombres, Madrid, Siglo XXI, 1980.

LARA IZQUIERDO, Pablo, Sistema aragonés de pesos y medidas: la metrología histórica aragonesa y sus relaciones con la castellana, Zaragoza, Guara, 1984.

LAWLOR, Robert, Sacred Geometry. Philosophy and practice, London, Thames \& Hudson, 1982 (reimpr. 2002).

MANIACI, Marilena, "Ricette di costruzione della pagina nei manoscritti greci e latini”, Scriptorium 49 (1995), pp. 16-51.

MANIACI, Marilena \& ORNATO, Ezio, "Intorno al testo. Il ruolo dei margini nell'impaginazione dei manoscritti greci e latini”, Nuovi Annali della Scuola Speciale per Archivisti e Bibliotecari 9 (1995), pp. 175-194. Republ. en La face cachée du livre médiéval. L'histoire du livre vue par Ezio Ornato, ses amis et ses collègues, Rome, Viella, 1997, pp. 457-471.

MASINI, Nicola; FONSECA, Cosimo Damiano; GERALDI, Edoardo; SABINO, Gabriela, "An algorithm for cumputing the original units of measure of medieval architecture", Journal of Cultural Heritage 5 (2004), pp. 7-15.

MATTÉI, Jean-François, "Nicomachus of Gerasa and the Arithmetic Scale of the Divine", in T. Koetsier \& L. Bergmans (eds.), Mathematics and the Divine: a Historical Study, Amsterdam, Elsevier, 2005, pp. 123-132.

MuZERELle, D., "Normes et recettes de mise en page dans le codex précarolingien", en A. BLANCHARD, Les débuts du codex. Actes de la journée d'étude organisée à Paris les 3 et 4 juillet 1985, Turnhout, Brepols, 1989, pp. 125-156.

Pellicer I BRU, Josep, Metrología. Diccionario descriptivo, Barcelona, Asociación numismática española, 2001.

PORTET, Pierre, "Remarques sur la métrologie carolingienne", Le Moyen-Âge, 5" série 1 (1991), pp. 5-24. 
RAND, Edward K., Studies in the Script of Tours. II. The Earliest Book of Tours, Cambridge, Mass., The Mediaeval Academy of America, 1934.

RoBInson, Andrew, Metrum: historia de las medidas, Barcelona, Paidós, 2007.

ROMÁN GARCíA, Ana María, "Notas para una bibliografía metrológica española", Boletín de Metrología 1 (1954), pp. 78-97; 2 (1955), pp. 59-76; 3 (1956), pp. 4970.

RoSARIVO, Raúl M., Divina proporción tipográfica, La Plata (Argentina), 1953.

RUIZ GARCÍA, Elisa, Introducción a la Codicología, Madrid, Fundación Germán Sánchez Ruipérez, 2002.

SNYDER, James, Medieval Art: Painting, Sculpture, Architecture, 4-14th Century, Upper Saddle River, N.J., Prentice Hall, 1989.

STEVICK, Robert D., “The Echternach Gospels' Evangelist Symbol Pages: Forms from 'The Two True Measures of Geometry", Peritia 5 (1986), pp. 284-308.

TAEGER, B., Zahlensymbolik bei Hraban, bei Hincmar und im 'Heliand'. Studien zur Zahlensymbolik im Frühmittelalter, München, C.H. Beck'sche Verlagsbuchhandlung, 1970.

TERRIEN, Marie-Pierre, "Religious architecture and Mathematics during the Late Antiquity", in T. KOETSIER \& L. BERGMAns (eds.), Mathematics and the Divine: a Historical Study, Amsterdam, Elsevier, 2005, pp. 147-160.

TOMASINI, María Celia, "El número y lo sagrado en el arte", Revista de Ciencia y Tecnología 4 (2003), pp. 53-70. [http://www.palermo.edu/ingenieria/ downloads/CyT\%204/CYT406.pdf, consulta 27/11/2012].

TORRENTS y MONNER, Antonio, Tratado de monedas, pesas y medidas antiguas y modernas de todos los países, Barcelona, Bayer Hnos., 1915.

TSCHICHOLD, Jan, "Consistent correlation between book page and type area", en Jan TSCHICHOLD, The form of the book. Essays on the morality of good design, Vancouver, Hartley \& Marks, 1991, pp. 36-63.

TSCHICHOLD, Jan, "Non-Arbitrary Proportions of Page and Type Areas", en A.S. OSLEY (ed.), Calligraphy and Paleography. Essays presented to Alfred Fairbank, London, Faber and Faber, 1965, pp. 179-191.

VINCENT, Auguste, "Notes de Mëtrologie. Les mesures locales dans les incunables", Revue Belge de Philologie et d'Histoire 5 (1926), pp. 955-966.

WITTHÖFT, Harald, Umrisee einer historischen Metrologie zum Nutzen der wirtschafts-und sozialgeschichtlichen Forschung: Mass und Gewicht in Stadt und Land Lüneburg, in Hansenraum und im Kurfürstentum/Königreich Hannover von 13. bis zum 19. Jahrhundert, Veröffentlichungen des MaxPlancks-Instituts für Geschichte 60/1, Göttingen, Vandenhoek \& Ruprecht, 1979.

ZAITSEV, Eugeny A., "The meaning of early medieval Geometry: from Euclid and Surveyors' manuals to Christian Philosophy”, Isis 90/3 (1999), pp 522-553.

ZUPKO, R.E., Italian Weights and Measures from the Middle Ages to the Nineteenth Century, Philadelphia, The American Philosophical Society, 1981. 